\title{
Juego de realidad alternativa para las matemáticas en educación superior desde la percepción estudiantil de las prácticas de enseñanza
}

\section{Alternate reality game for mathematics in higher education from the student perception of teaching practices}

DOI: http://dx.doi.org/10.17981/cultedusoc.10.2.2019.10

Recibido: 09/08/2019 Aceptado: 15/11/2019

Diego Darío López-Mera

Institución Universitaria Antonio José Camacho (Colombia) diegodario21@gmail.com

Brayan Camilo Hernández-Montoya

Institución Universitaria Antonio José Camacho (Colombia) bchernandez@profesores.uniajc.edu.co

Sandra Esther Suarez-Chavez

Institución Universitaria Antonio José Camacho (Colombia) ssuarez@admon.uniajc.edu.co

Ana Catalina Archila-Gutiérrez

Institución Universitaria Antonio José Camacho (Colombia) acarchila@admon.uniajc.edu.co

Eider Hernán Pérez-Rojas

Institución Universitaria Antonio José Camacho (Colombia) eperez@admon.uniajc.edu.co

\section{Sandra Viviana Osorno-Taborda}

Institución Universitaria Antonio José Camacho (Colombia) sandravivianaosorno@hotmail.com

Para citar este artículo:

López-Mera, D., Hernández-Montoya, B.,Suarez-Chavez, S.,Archila-Gutiérrez, A.,Pérez-Rojas, E. y Osorno-Taborda, S. (2019). Juego de realidad alternativa para las matemáticas en educación superior desde la percepción estudiantil de las prácticas de enseñanza. Cultura, Educación y Sociedad, 10(2). 121-134. DOI: http://dx.doi.org/10.17981/cultedusoc.10.2.2019.10

Resumen

Este artículo analiza las percepciones y preferencias que tienen los estudiantes de primer semestre de los programas tecnológicos de las facultades de Ingeniería y Ciencias Empresariales de la Institución Universitaria Antonio José Camacho - Colombia (UNIAJC), sobre las estrategias de enseñanza de las matemáticas aplicadas por los profesores en los cursos de Matemáticas 1. La indagación corresponde a una iniciativa pedagógica centrada en el desarrollo de un Juego de Realidad Alternativa (Alternate Reality Game o ARG) que permita favorecer el aprendizaje de las matemáticas de una forma lúdica y poco convencional. Para el desarrollo del ARG y adaptabilidad al contexto educativo, se consulta en los estudiantes sus gustos literarios, cinematográficos, videojuegos y problemáticas sociales de mayor importancia, estrechamente relacionados a su contexto. Los resultados en atención a las variables trabajadas permiten elaborar una primera estructura narrativa del ARG. Se concluye que el diseño y desarrollo de una propuesta educativa basada en un ARG, siempre debe estar relacionada con las necesidades y percepciones del sujeto del aprendizaje.

Palabras clave: aprendizaje basado en juegos; educación matemática; estrategias de enseñanza; juego de realidad alternativa; percepción estudiantil

\section{Abstract}

This article analyzes the perceptions and preferences that first-semester students have of the technological programs of the Engineering and Business Sciences faculties of the Antonio José Camacho University Institution - Colombia (UNIAJC for its acronym in Spanish), on the teaching strategies of mathematics applied by students. Teachers in Mathematics courses 1 . The inquiry corresponds to a pedagogical initiative focused on the development of an Alternate Reality Game (ARG) that allows the learning of mathematics in a playful and unconventional way. For the development of the ARG and adaptability to the educational context, students are consulted on their literary, cinematographic, videogame and social problems of greater importance, closely related to their context. The results in attention to the variables worked allow to elaborate a first narrative structure of the ARG. It is concluded that the design and development of an educational proposal based on an ARG must always be related to the needs and perceptions of the learning subject.

Keywords: alternate reality game; game-based learning; mathematics education; student perception; teaching strategies 


\section{INTRODUCCIÓN}

Las transformaciones sociales del siglo XXI han generado tensiones en la educación tradicional, por las renovaciones de las prácticas sociales e ideológicas y la incursión de las tecnologías de la información y comunicación en la vida diaria. Esta dinámica altera el espacio y el tiempo de los aprendizajes, y la importancia de ellos en el ámbito educativo. Los estudiantes demandan formas alternativas de aprender para no caer en un "desvanecimiento del sentido de la educación" (Coll, 2009, p. 4), el cual es atizado por los contenidos de aprendizaje que poco se relacionan con la cotidianidad del estudiante, donde su función o utilidad se pone en entredicho en la vida práctica del mismo.

Es importante, entonces, repensar dentro de la educación, formas de enseñanza alternativas que involucren e incentiven la motivación del estudiante; y el juego puede ser una de estas formas. Los juegos siempre han acompañado a la humanidad a lo largo de su desarrollo (Huizinga, 1998). Inclusive, están presentes desde las primeras etapas de vida del ser humano.

Pero para que el juego pueda generar un aprendizaje, y en este caso para su utilización en un curso, debe tener la connotación de «juego serio» en el que la educación es el principal objetivo, en lugar del sólo entretenimiento (Chen y Michael, 2006; Djaouti, Álvarez y Jessel, 2011; García, Vargas, Genero y Piattini, 2014). Se puede comprender que los juegos, propiamente en educación, deben contar necesariamente con objetivos de aprendizaje claros para la adquisición de la enseñanza por parte del sujeto del aprendizaje. Si, por el contrario, el juego tiene una connotación de juego libre, difícilmente podrá vincularse al contexto educativo, por su carencia en la finalidad de los propósitos de la enseñanza que debería tener para su realización. Si se articulan los propósitos de enseñanza, podría el juego ser un mecanismo de aprendizaje significativo, porque desarrolla y compromete el pensamiento creativo, emocional, la solución de problemas y el lenguaje del estudiante.

Ahora bien, el juego, a su vez, se puede emplear en distintas áreas del conocimiento, por ejemplo, en las matemáticas. Al respecto, Oldfield (1991) refiere la siguiente definición de juego como juego matemático:

1. La actividad involucra: a) Un desafío contra una tarea o uno o más oponentes. b) $\mathrm{O}$ una tarea común que debe abordarse ya sea solo o, más comúnmente, en conjunción con otros.

2. La actividad se rige por un conjunto de reglas y tiene una estructura clara subyacente a las mismas.

3. La actividad normalmente tiene un final distinto.

4. La actividad tiene objetivos matemáticos y cognitivos específicos (citado por González, Molina y Sánchez, 2014, p. 114).

Si se observa el enunciado anterior, la actividad que compone el juego matemático contiene un desafío o reto que puede ser un eje motivador para el estudiante, porque implica superar lúdicamente una tarea compleja con respecto al área de la matemática. Si se ubica esta lógica a la aplicabilidad tecnológica, su utilidad, por ejemplo, podría estar representada en Geogebra, una herramienta tecno-pedagógica que puede ser utilizada como una estrategia lúdica para el aprendizaje matemático mediante el trabajo colaborativo de estudiantes y profesores (Gülseçen, Kararaş \& Koçoğlu, 2012; Lit, 2013; Olsson, 2017), o en otras como Derive (Pulido, 2002; Dominici y González, 2010) o Scilab (Medina y Rubio, 2013). 
Sin embargo, la lúdica y el juego en la práctica de enseñanza de las matemáticas no debe depender únicamente de lo tecnológico, también puede considerar espacios físicos o ambientes pensados desde lo social, donde el estudiante se relaciona y teje sus sentidos de vida con otros. Talavera y Marín (2015), destacan la importancia de integrar la tecnología, al ciclo didáctico desde la posibilidad de crear espacios virtuales de aprendizaje. Aun cuando se asume el aprendizaje tecnológico (Cárdenas y Ángulo, 2016) centrado en la mediación y adaptación de las tecnologías en el proceso educativo, es necesario, pensar en una pedagogía de enseñanza que involucre dos características fundamentales (juego y tecnología), teniendo en cuenta las necesidades de aprendizaje y los sentidos de vida de los estudiantes, situación que en principio parece compleja. $\mathrm{Al}$ respecto,

Precisamente, los juegos de realidad alternativa tienen dichas características, ya que son ficciones interactivas e inmersivas que cuentan con los tres elementos: una serie de retos, una narrativa subyacente y una comunidad colaborativa, y aunque la integración de estos elementos se facilitan en la Web, muchos retos tienen lugar en el mundo real y pueden ser asumidos por los jugadores de forma colaborativa, preferiblemente (Askwith, 2006; Whitton y Hollins, 2008; De Beer, 2015; Palmer y Petroski, 2016). Así que en la ejecución de un ARG confluyen "experiencias interactivas inmersivas, donde los jugadores de forma colaborativa localizan claves, organizan información dispersa y resuelven enigmas para avanzar en la narrativa que combina tanto el entorno real como el online" (Piñeiro y Costa, 2015, p. 3). Estas características hacen que los ARG tengan un gran potencial para ser usados en la educación, con el fin de pro- porcionar actividades colaborativas útiles y motivadoras que logren contextualizar el aprendizaje (Goodlander, 2008; Connolly, Stansfield y Hainey, 2011; Bonsignore et al, 2012; Waddington, 2013). De este modo, los ARG ya se están desarrollando y utilizando con diversos propósitos formativos (Fujimoto, 2010; Dondlinger \& McLeod, 2015; Jerrett, 2016). Algunos se diseñan para ser jugados en días, semanas o hasta meses, y puesto que implican la integración de tecnologías web (blogs, wikis, redes sociales online y entornos para compartir recursos) proporcionan a los usuarios la experiencia de diferentes tecnologías (Whitton y Hollins, 2008).

De tal modo que de acuerdo a la necesidad de ir avanzando en la innovación y mejoramiento académico dentro del aula con los estudiantes del curso Matemática 1 de la UNIAJC, se conforma un grupo multidisciplinar para el diseño y desarrollo de un ARG. En principio, se lleva a cabo una indagación en los estudiantes sobre sus percepciones y gustos académicos, relacionados a sus intereses de aprendizaje frente a las formas de enseñanza de las matemáticas. La información recogida posibilita la elaboración preliminar de un ARG para la enseñanza de las matemáticas.

\section{Prácticas de enseñanza}

Toda actividad que implique una intencionalidad de enseñanza debe estar necesariamente condicionada a un modelo pedagógico; pero antes de entrar en materia sobre el modelo pedagógico utilizado para la construcción del ARG, es necesario tener un acercamiento a lo que es la práctica de enseñanza en sí. De Landsheere (1976) refiere que "las prácticas de enseñanza son el punto de encuentro del saber teórico 
con su campo de aplicación" (p. 37); Montero (1987), en esta misma línea, señala la definición: "las prácticas de enseñanza son experiencias planificadas para capacitar al futuro profesor en la utilización de los conocimientos pertinentes acerca de su función en situaciones concretas de enseñanza-aprendizaje" (p. 41).

¿Qué requiere, entonces, la enseñanza? Un profesor que planifique su método de enseñanza y, por supuesto, las actividades a realizar después de la enseñanza que, es conocido también como "enseñanza preactiva" (Pérez, Zabalza y Cid, 2009). Pero como se mencionó anteriormente la práctica de enseñanza requiere de un modelo pedagógico. En el caso propio de la investigación se utiliza el modelo del enfoque socioformativo (Tobón, González, Nambo y Vázquez, 2015), el cual se basa en un marco por competencias, fundamentado en el pensamiento sistémico y complejo. Según Tobón, Pimienta y García (2010) este énfasis basado en el concepto de competencias consiste en la "interpretación, argumentación y resolución de problemas de contexto externo" (p. 9) y la "formación e idoneidad y compromiso ético en todas las competencias" (p. 9); de tal modo que en el estudiante y en el currículo se "busca afrontar los retos personales, institucionales y del contexto externo, actuales y futuros" (p. 9).

De esta forma, el enfoque socioformativo no está sólo pensado para la resolución de problemas de contexto interno, sino también externo; es decir, el estudiante no solo debe mostrar su saber hacer en su contexto cotidiano, sino además en otros contextos alternos que requieran su desenvolvimiento oportuno.

Para el cumplimiento de dicho objetivo, el profesor debe generar una enseñanza donde se garantice el desarrollo de la competencia por parte del estudiante. Se requiere de estrategias para contribuir a ese saber hacer. Sin embargo, una cosa es que el profesor considere que su práctica de enseñanza es la más indicada o pertinente, y otro asunto es lo que perciben los estudiantes sobre su práctica de enseñanza. Por tal razón, indagar sobre las percepciones de los estudiantes acerca de la práctica de enseñanza de las matemáticas, fortalece pedagógicamente las actividades a emplear en el ARG.

Pero ¿qué se podría entenderse por percepción? Es sin duda, la herramienta fisiológica, cognitiva y simbólica que posibilita conocer e interpretar el mundo externo, el cual se va nutriendo paulatinamente de las experiencias de vida del sujeto. Si se abordan dos instancias, tanto la fisiológica como la cognitiva, se puede afirmar que el acto perceptivo está sujeto a dos condiciones. La primera relacionada con la necesidad de contar con un cuerpo sensitivo receptor de estímulos. La otra condición está en que ese cuerpo sensitivo requiere de estímulos externos (información) a ser procesados en el cerebro y crear así una imagen. De este modo, la percepción puede definirse como "el proceso mental de interpretar y dar significado a la sensación de un objeto determinado" (Kelly, 1982, p. 71). En el marco propio de esta investigación, la percepción está suscrita a la interpretación y significación que les dan los estudiantes a sus procesos de enseñanza y aprendizaje de las matemáticas; así como todos los factores asociados a dichos procesos. Para conocerlos, se presenta a continuación, el método de investigación utilizado en el desarrollo de la investigación, el cual además presenta una caracterización de la población estudiada en género y rango de edad. Las categorías de análisis están descritas en el contenido del método. 


\section{MÉToDo}

Para el análisis de los intereses y percepciones de los estudiantes, se aplica una encuesta a 76 estudiantes de primer semestre con un rango de edad entre los 16 y 33 años, identificando un promedio de edad de 21 años y una desviación estándar de 9.6 años. En cuanto a la participación por género se tiene el $36.8 \%(\mathrm{n}=28)$ del género femenino y el $63.2 \%(n=48)$ del masculino. Sobre la distribución por estratos de la muestra, se tiene que un $22 \%$ pertenecen al estrato 1; el $36 \%$, al estrato 2 y el otro $36 \%$, al estrato 3 ; quedando un $6 \%$, en estrato 4 y 5 (Figura 1).

La encuesta está conformada por 22 preguntas, clasificadas en las siguientes categorías de análisis: cotidianidad, metodología de enseñanza de las asignaturas de las matemáticas y usos en medios de comunicación. Las preguntas que se exponen en los resultados son preguntas que contribuyen mayormente al diseño de la ARG, las demás están orientadas al acceso que tienen los estudiantes al Internet, por tal motivo no se abordan en los resultados.
La metodología de análisis se desarrolla de la siguiente forma:

1. Tomando de referencia para elaborar la encuesta lo propuesto por Tobón, Pimienta y García (2010) sobre los componentes principales del enfoque socioformativo, teniendo en cuenta caracterizar los sentidos que otorgan los estudiantes a las problemáticas de su contexto cotidiano.

2. Las preguntas y criterios de análisis son elaboradas con un equipo investigador multidisciplinar (ingenieros, psicólogos, estadísticos y diseñadores). Las características de los criterios de análisis se construyen teniendo en cuenta el enfoque socioformativo y el de pensamiento complejo, así mismo los intereses de los estudiantes frente a la enseñanza de las matemáticas y accesibilidad al Internet. Esto último se toma en cuenta porque el diseño de los ARG está también sustentado por el uso tecnológico. Si los estudiantes no cuentan con acceso a Internet, el equipo investigador debe asumir otro tipo de dinámicas para la creación de los retos de la historia del ARG.

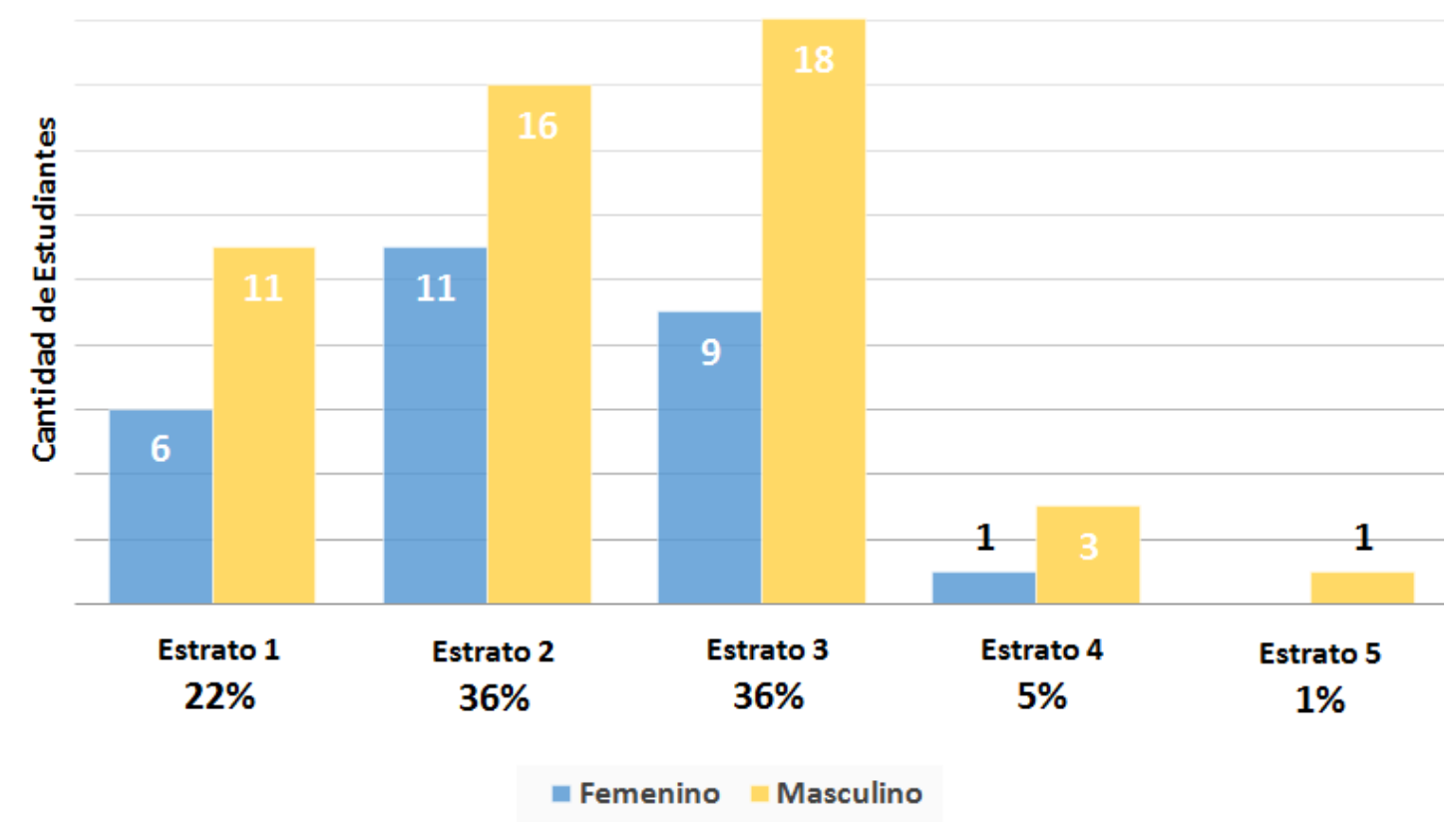

Figura 1. Distribución de los estudiantes encuestados según el nivel socioeconómico y género. Fuente: elaboración propia. 


\section{RESUlTAdos}

Se crean categorías para agrupar las respuestas de los estudiantes de acuerdo con las similitudes y diferencias de sus respuestas. De este modo se obtiene un número grande de categorías que luego son agrupadas en una cantidad mucho menor de subcategorías para facilitar el respectivo análisis de la información suministrada por los estudiantes. Las respuestas se agrupan de acuerdo con la principal categoría que el estudiante expresa sobre cada una de las preguntas.

Para la pregunta «¿cuáles son las actividades desarrolladas por un profesor que más te motivan a aprender?», los estudiantes manifiestan que el trabajo en grupo en clase es una de las actividades preferidas durante su proceso de aprendizaje, así como clases dinámicas y desarrollo de ejercicios prácticos (Figura 2). De igual modo, los estudiantes dan importancia a la explicación práctica y amena del profesor.

Para los "grupos de trabajo en clase», los estudiantes prefieren actividades que gene- ran interacción y debates entre los compañeros, en las cuales adicionalmente logren despejar dudas sobre el tema. También recalcan clases dinámicas como espacio en donde se realicen actividades no rutinarias, creativas y con salidas pedagógicas. Además prefieren realizar ejercicios prácticos en clase con aplicación temática, prácticas de laboratorio y la resolución de talleres para validar los procedimientos y respuestas, esto sumado a la forma amena de explicar en donde se valore el logro de objetivos de aprendizajes.

En la validación de la pregunta «¿cuáles son las actividades desarrolladas por un profesor que menos te motivan a aprender?», los estudiantes refieren que menos les gusta es una clase con el uso de mucha teoría, así como la cantidad de temas abarcados por los profesores (Figura 3). Es también importante para el estudiante la forma de explicar del profesor, pues perciben que afecta mucho su proceso de aprendizaje. La clase monótona y colocar muchas tareas también lo ven como malas prácticas en la pedagogía en clase.

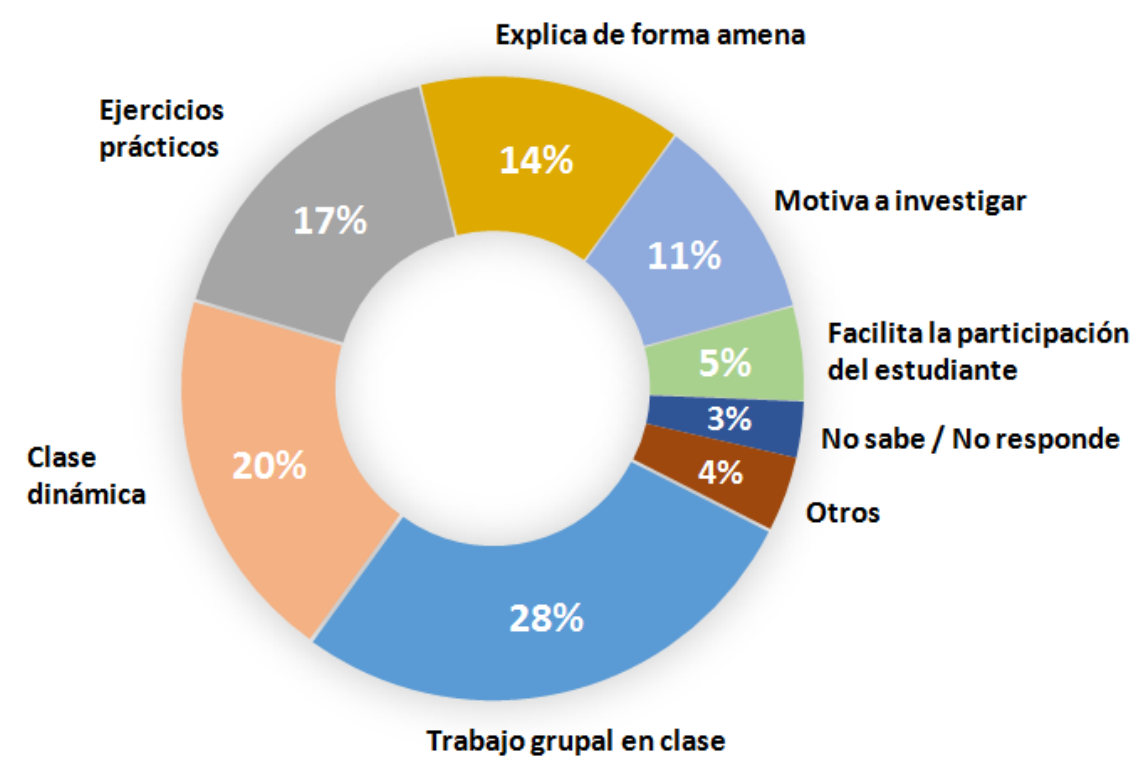

Figura 2. Categorías para la pregunta del instrumento: ¿cuáles son las actividades desarrolladas por un profesor que más te motivan a aprender? Fuente: elaboración propia. 


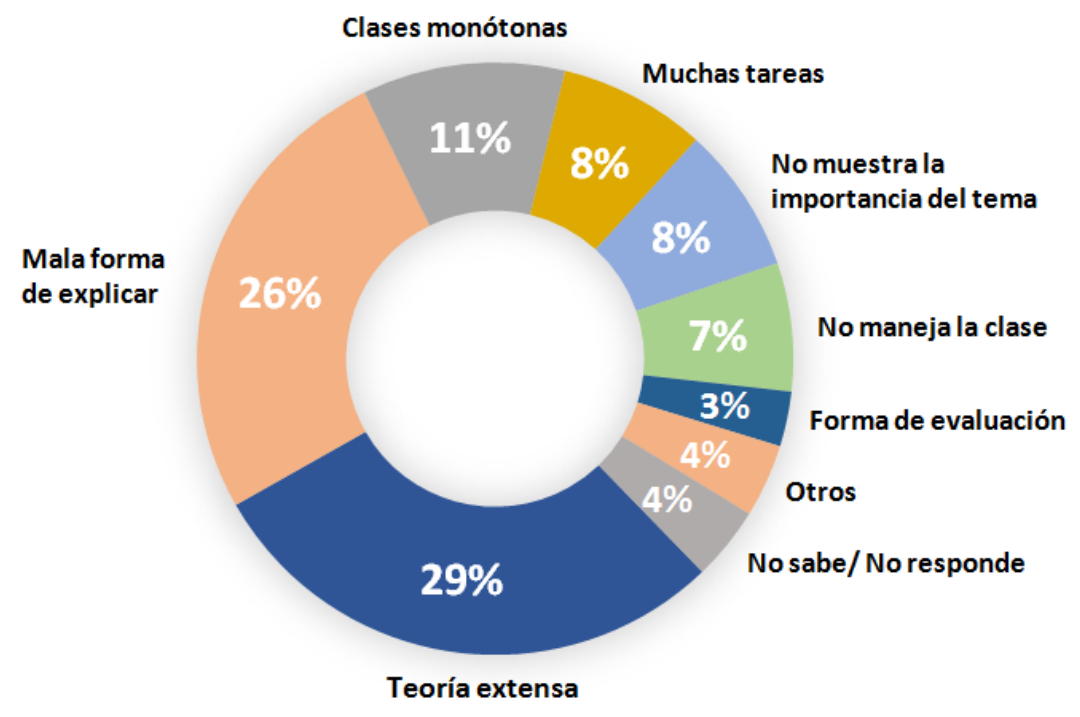

Figura 3. Categorías para la pregunta del instrumento:

¿cuáles son las actividades desarrolladas por un profesor que menos te motivan a aprender? Fuente: elaboración propia.

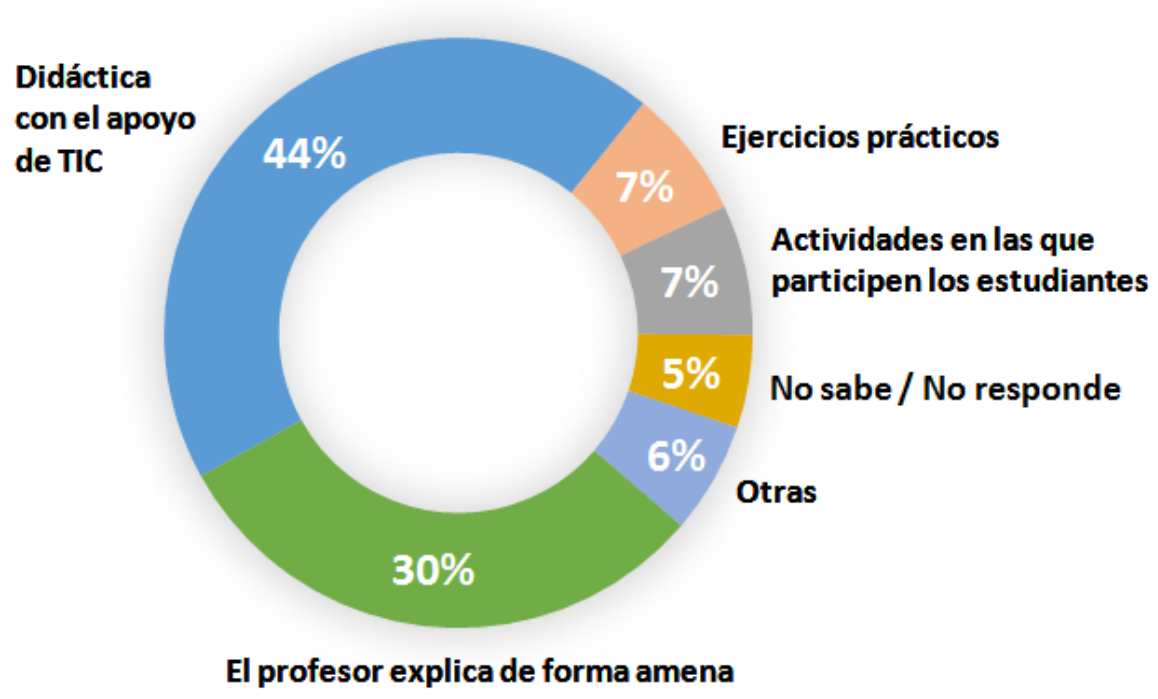

Figura 4. Categorías para la pregunta del instrumento:

¿qué experiencias recuerdas que hayan sido más agradables y efectivas para tu aprendizaje?

Fuente: elaboración propia.

Con respecto al punto de «Teoría Extensa» en la Figura 3, los estudiantes manifiestan que estar en clase recibiendo mucha teoría, muchos temas y sin ejercicios prácticos, lo califican en un $29 \%$ de las actividades que menos les gusta. También desaprueban las clases donde el Profesor explica de manera acelerada, sin importarle que el estudiante entienda o peor aún, mostrando una actitud inadecuada ante una pregunta sobre lo explicado.
Para la pregunta «de la forma de enseñanza de las matemáticas, ¿qué experiencias recuerdas como agradables y efectivas para tu aprendizaje?», los estudiantes manifestaron que la didáctica es lo más agradable y efectivo para su aprendizaje, seguido de la forma amena de explicar (Figura 4). También es importante el desarrollo de ejercicios prácticos y dar participación a los estudiantes. 


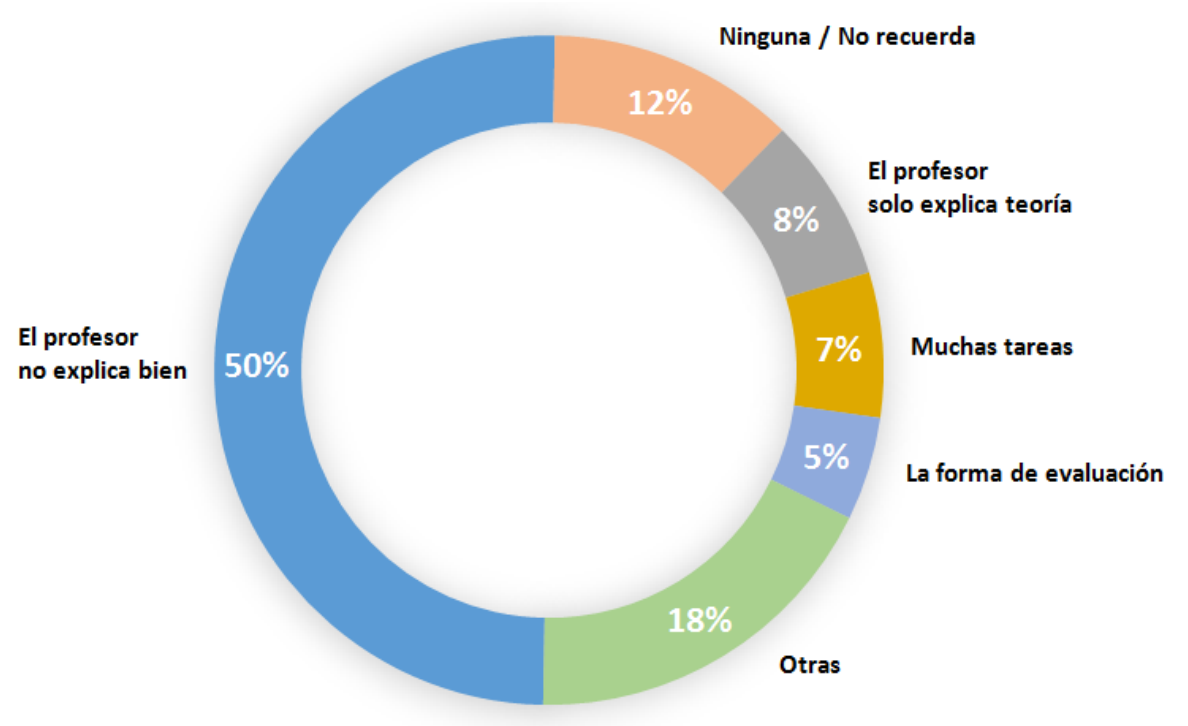

Figura 5. Categorías para la pregunta del instrumento:

¿qué experiencias recuerdas que hayan sido menos agradables y efectivas para tu aprendizaje? Fuente: Elaboración propia.

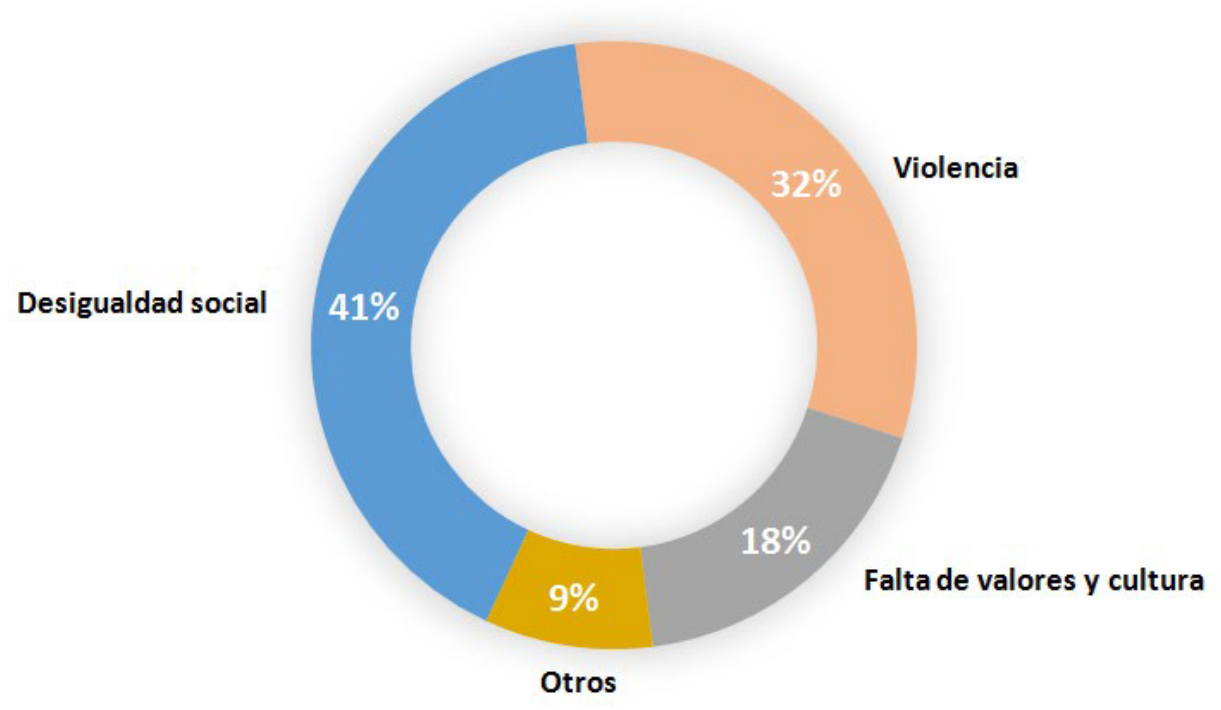

Figura 6. Categoría pregunta del instrumento:

si tuvieras la oportunidad de resolver una problemática a nivel social, ¿cuál resolverías?

Fuente: Elaboración propia.

Validando con la pregunta, ¿qué experiencias recuerdas como menos agradables y efectivas para tu aprendizaje?», los estudiantes afirman que lo menos agradable en el proceso de aprendizaje en matemáticas es que el profesor no explique bien, donde solo seexplique teoría (Figura 5). En tanto, un $12 \%$ no recuerda o no tuvo una mala experiencia en la clase de matemáticas.

También se indagó sobre la dimensión social en relación al aprendizaje. La Figura
6 muestra los resultados a la pregunta «si tuvieras la oportunidad de resolver una problemática a nivel social, ¿cuál resolverías?». Los estudiantes evidencian una preocupación por situaciones de desigualdad social, violencia y falta de valores y cultura, en las que no sólo las relacionan con casos de inseguridad, intolerancia y pobreza; sino, además, con la corrupción y la falta de cobertura en el sistema educativo. 


\section{DiscUSIÓN}

Los resultados indican en la muestra analizada $(\mathrm{N}=76)$ que el proceso de elaboración del ARG debe considerar en el diseño de sus retos, ejercicios prácticos y dinámicos desde un trabajo colaborativo que pueda llevar a cabo el estudiante con sus pares. Asimismo, los retos deben implicar un ejercicio práctico para que el estudiante dinamice su aprendizaje. Lo encontrado, se puede relacionar con la pretensión educativa del enfoque socioformativo, dirigido a que el sujeto del aprendizaje pueda afrontar retos personales e institucionales desde el hacer práctico del contexto (Tobón, Pimienta y García, 2010).

Es tal la urgencia del sentido y ejercicio práctico del aprendizaje, que la mayoría de los estudiantes refirieron sentirse desmotivados, cuando están involucrados en clases donde se imparte una teoría extensa y más aún cuando esa teoría se vuelve más compleja con las dificultades que tiene el profesor para hacerse explicar. En este orden de ideas, la práctica de enseñanza de los profesores se puede ubicar en una práctica tradicional, contrario a su carácter novedoso, en el sentido de proporcionar un aprendizaje con habilidades experimentales y cognitivas "que surjan como consecuencia fluida de la relevancia y la significatividad de los tópicos de la naturaleza de la ciencia, más que como motivo primario del aprendizaje" (Torres, 2010, p. 138). De continuar con dicha práctica de enseñanza, se incentivaría el "desvanecimiento del sentido de la educación” referido por Coll (2009).

Por tanto, el diseño del ARG no sólo tiene el desafío de generar un proceso de enseñanza de las matemáticas en el estudiante, correspondiente al contexto donde se desenvuelve, sino también debe pensarse desde su diseño, en dos aspectos clave referidos los estudiantes: la didáctica y la desigualdad social. La primera deberá responder a la pregunta: ¿cómo hacer posibles los procedimientos de la enseñanza? Una cuestión que atañe a la etiología de la didáctica (Díaz, 2002). Y en respuesta, el procedimiento de enseñanza a utilizar desde el ARG, es su historia que tendrá como tema central la desigualdad social (segundo aspecto clave expresado por los estudiantes). Es decir, la historia del ARG se realiza con los contenidos enunciados por los estudiantes, correspondientes a sus sentidos de vida y que posibilitan vincular sus motivaciones para el ejercicio de la enseñanza y aprendizaje de las matemáticas; más aún, cuando son problemáticas sociales vinculadas día a día en su contexto. De esta forma el proceso del ARG responde a las percepciones generadas por los estudiantes.

\section{Boceto preliminar de la construcción del ARG "El plan de Gauss»}

Con lo mencionado en el anterior apartado, se diseña el juego de realidad alternativa «El plan de Gauss», narra la historia de tres estudiantes de primer semestre de la UNIAJC de diferentes carreras, quienes coinciden en el curso de matemáticas 1 . En esta historia tanto los personajes como los jugadores (estudiantes del curso) se les asigna la misión de buscar a un personaje desaparecido misteriosamente. Durante la búsqueda, los jugadores se enfrentan a retos matemáticos, en su mayoría para ser jugados de forma colaborativa y contextualizados con casos de corrupción, inequidad social, inseguridad y falta de ética en temas relacionados con la salud, el medio ambiente y la nutrición infantil, entre otros.

Se estructura un relato desarrollado en el nivel socioeconómico preponderante de los estudiantes de la UNIAJC, es decir, estratos 1, 2 y 3 . En cuanto a las matemáticas, 
se seleccionan los tres primeros conceptos estudiados en el curso de matemáticas 1: funciones lineales, funciones cuadráticas y sistemas de ecuaciones lineales. Este ARG se planea para ser jugado durante la primera mitad del curso. Específicamente, su duración es de dos meses y las actividades, es decir los «retos», se jugarán en el transcurso de ocho clases, una clase por semana. Se diseñó un reto por clase y otros más que son puestos como tareas.

De este modo se contemplan los siguientes medios y contenidos tecnológicos y de multimedia para el desarrollo del ARG «El plan de Gauss":

- Se crean blogs para los personajes principales a utilizar como medios para que los jugadores conozcan la historia. También se crea un grupo de Facebook que uno de los personajes utiliza para comunicarse con los jugadores.

- Se realiza un videotutorial para explicar el concepto de relación matemática, una nota periodística en vídeo para informar sobre las actividades de uno de los personajes del ARG y varios cortometrajes en animación 2D para contar momentos de la historia.

- Se realizan relatos interactivos denominados "novelas visuales" como medios alternativos para contar la historia y acceder a los retos.

- Se crean dos artefactos que existen tanto en el mundo ficcional como en el mundo del jugador: una gaceta financiera digital y un sitio web de una convocatoria de emprendimiento, patrocinada por uno de los personajes.

- Se elaboran retos matemáticos para diversos medios y plataformas. Unos para ser jugados en clase con la participación y acompañamiento del profesor, y otros que son puestos como tareas.
A continuación, se hace una descripción general de los retos diseñados para el ARG:

1. En la historia del ARG, uno de los personajes desaparece y los estudiantes tienen la misión de ayudar en su búsqueda, utilizando un listado de direcciones de los lugares que frecuentaba el personaje desaparecido. Por medio de una pantalla que muestra un plano cartesiano que simula el mapa de una ciudad y un sensor de detección de movimientos, los estudiantes conforman grupos para ubicar en el menor tiempo posible las direcciones del listado, en el cual las calles y las carreras son las coordenadas $x$ y $y$, respectivamente.

2. Uno de los personajes del ARG sigue desaparecido y los estudiantes tienen la misión de investigar a los demás personajes en busca de un sospechoso o una pista. Para tal propósito, revisan sus blogs y redes sociales, y así encontrar características comunes que los relacionen y las puedan representar mediante diagramas de Venn o planos cartesianos.

3. Uno de los personajes principales del ARG es un empresario que crea una convocatoria en busca de jóvenes talentosos y emprendedores que quieran ser parte de sus negocios. En esta convocatoria, los estudiantes deben superar tres retos matemáticos, donde el primero plantea una situación, en la cual mediante evaluación de funciones se debe elegir la mejor decisión de inversión en un proyecto de un producto alimenticio.

4. En el ARG, un personaje que está en la actividad política denuncia un caso de corrupción en el que se roba dinero estatal con un programa que lanza el Gobierno de apoyo económico no reembolsable para los pequeños agricultores. 
Los estudiantes tienen la misión de calcular de cuánto asciende este robo.

5. En el ARG, uno de los personajes, quien trabaja para pagarse los estudios en la universidad, pasa por una mala situación económica. Los estudiantes tienen la misión de ayudarle a determinar cómo se distribuyen sus gastos e ingresos, y aportarle datos que le sirvan para analizar su situación financiera mediante el concepto de función lineal.

6. El jefe del personaje que pasa por un mal momento económico le propone hacer un fraude en el negocio donde trabajan. Los estudiantes analizan la situación, aplicando el concepto de función lineal.

7. El segundo reto en la convocatoria del personaje empresario consiste en evaluar un problema que se presenta en la inclinación de las escaleras en varios colegios. Mediante el concepto de pendiente de la línea recta, los estudiantes descubrirán malos manejos de recursos y casos de corrupción.

8. En el ARG, uno de los personajes principales tiene a su mamá enferma. En este reto, los estudiantes descubren la falta de ética en el tema de la salud, al realizar cálculos donde se demuestra que los medicamentos proporcionados a la mamá del personaje, no cumplen con los estándares de calidad requeridos para contribuir al alivio de su enfermedad.

9. En el tercer reto de la convocatoria del personaje empresario, los estudiantes deben analizar un caso de corrupción relacionado con los desayunos escolares, mediante sistemas de ecuaciones lineales.

10. Uno de los personajes sigue desaparecido y una misteriosa llamada telefónica aporta nuevas pistas. Los estudiantes tienen la misión de descubrir un lugar en especial en el que se encontraba el personaje antes de desaparecer, por medio del método gráfico y la solución matemática de un sistema de ecuaciones lineales de $2 \times 2$.

11. En este reto, los estudiantes analizan un estudio realizado sobre el comportamiento de un nuevo producto en el mercado, dado por una función cuadrática.

12. Uno de los personajes decide crear un negocio propio, para solucionar sus problemas económicos. Los estudiantes analizarán la viabilidad determinando la ecuación cuadrática de la función de ingresos y la incidencia que tiene la concavidad en el comportamiento de los ingresos a través del tiempo.

13. Este es el reto final y consiste en participar en un concurso de preguntas y respuestas, con respecto a todos los conceptos matemáticos tratados en el ARG.

\section{Conclusiones}

De acuerdo con los resultados expuestos con anterioridad, se puede considerar que el diseño y desarrollo de una propuesta educativa basada en un ARG, siempre debe estar relacionada con las necesidades y percepciones del sujeto del aprendizaje. Es decir, su desarrollo operativo debe considerar y reconocer los sentidos de vida académica y social del estudiante para que el contenido del ARG y su puesta en marcha tenga un impacto pedagógico mucho más significativo en el estudiante.

En el ARG resultante de esta investigación, todo se contextualizó a las situaciones problemas de la cotidianidad y entorno del estudiante de la UNIAJC; así que la desigualdad social, uno de los criterios más señalados por ellos, estuvo presente en la historia y los retos del juego. 
Otro aspecto importante para destacar es la urgencia y el llamado que hacen los estudiantes para que las formas tradicionales de enseñanza de las matemáticas incorporen aspectos pedagógicos más prácticos y dinámicos; es decir, que se articulen a enfoques como el socioformativo donde se involucre el trabajo colaborativo. El sujeto del aprendizaje coloca siempre un desafío al profesor en su práctica de enseñanza, le demanda un saber decir que vaya más allá de la teoría, para ubicarse mucho más en un saber práctico. El profesor debe saber cómo decir lo que quiere decir y requerirá, entonces, de una didáctica de enseñanza distinta para superar clases monótonas y carentes de sentido.

El ARG es una apuesta alternativa y didáctica para mitigar dicha situación, no sólo de las matemáticas, sino también de otras disciplinas y ciencias. El ARG apropia una historia con desafíos y sentidos pedagógicos del sujeto del aprendizaje, pero también hace lo mismo con recursos tecnológicos como apoyo para el alcance de los objetivos de aprendizaje que se proponga alcanzar el profesor en el aula y por fuera de ella.

La enseñanza no puede ser un asunto que apague el interés del estudiante, por el contrario, debe promover la inquietud y la motivación por aprender y saber-hacer en contexto. Si el promotor y orientador de la enseñanza omite los sentidos de vida que se tejen alrededor de la necesidad educativa y social de los estudiantes, quedará impartiendo contenidos precarios de sentido, donde la práctica de enseñanza, únicamente será emotiva para el profesor; en un circunloquio que imposibilita alcanzar objetivos pedagógicos significativos para el sujeto del aprendizaje.

\section{REFERENCIAS}

Askwith, I. (2006). This is not (just) an advertisement: Understanding alternate reality games. Convergence Culture Consortium, Comparative Media.

Bonsignore, E., Hansen, D., Kraus, K., Ahn, J., Visconti, A., Fraistat, A. y Druin, A. (2012). Alternate Reality Games: platforms for collaborative learning. International Journal of Learning and Media, 4(1), 25-54. http://doi.org/10.1162/ IJLM a 00086

Cárdenas, N. y Angulo, F. (2016). Análisis de las dimensiones de adaptación, mejoramiento e innovación en los procesos de aprendizaje tecnológico. Cultura Educación y Sociedad, 7(2), 139-149. Recuperado a partir de https://revistascientificas.cuc.edu.co/culturaeducacionysociedad/article/view/1468

Coll, C. (2009). Enseñar y aprender en el siglo XXI: el sentido de los aprendizajes escolares. En, A. Marchesi, J. Tedesco y C. Coll (Coords.), Calidad, equidad y reformas de la enseñanza. Madrid: OEI/Fundación Santillana.

Connolly, T., Stansfield, M. y Hainey, T. (2011). Alternate reality game for language learning: ARGuing for multilingual motivation. Computers \& Education, 57(1), 1389-1415. http://doi.org/ bhs9ng

Chen, S. y Michael, D. (2006). Serious games: Games that educate, train, and inform. Canada: Thomson.

De Beer, K. (2015). Analysing alternate reality games based on game design theory to propose a conceptual framework. (Tesis de maestría). University of Pretoria. Sudáfrica. Recuperado de http://hdl.handle.net/2263/57176

De Landsheere, V. (1976). Définir les objectifs de l'éducation. P.U.F. Education. 
Díaz, F. (2002). Didáctica y currículum: un enfoque constructivista. Cuenca: Universidad de Castilla - La Mancha.

Djaouti, D., Álvarez, J. \& Jessel, J. P. (2011). Classifying serious games: the G/P/S model. Handbook of research on improving learning and motivation through educational games: multidisciplinary approaches, 2(1), 118-136. http://doi.org/ deverp

Dominici, J. y González, O. (2010). Propuesta didáctica para la enseñanza del tema funciones a través de la utilización de estrategias metacognitivas y el uso del Derive. Unión. Revista Iberoamericana de Educación Matemática, 22(1), 91-107.

Dondlinger, M. y McLeod, J. (2015). Solving real world problems with alternate reality gaming: student experiences in the global Village Playground capstone course design. Interdisciplinary Journal of Problem-Based Learning, 9(2). http:// doi.org/cqfa

Fujimoto, R. (2010). Designing an educational Alternate Reality Game. Shoyu Learning Solutions. Available from http://www.shoyu.com/education/Research_DesigningAnEducationalARG. pdf

Huizinga, J. (1998). Homo ludens. (1938). Madrid: Alianza.

Jerrett, A. (2016). Using alternate reality game to teach information literacy. ( $\mathrm{Te}$ sis de maestría). University of Pretoria, Sudáfrica. Recuperado de https://repository.up.ac.za/handle/2263/57484

García, L., Vargas, J., Genero, M. y Piattini, M. (2014). ¿Contribuye el uso de juegos serios a mejorar el aprendizaje en el área de la informática? Jornadas de Enseñanza Universitaria de la Informática. Oviedo: Universidad de Oviedo. Recuperado de http://hdl.handle. net/2099/15478
González, A., Molina, J., y Sánchez, A. (2014). La matemática nunca deja de ser un juego: investigaciones sobre los efectos del uso de juegos en la enseñanza de las matemáticas. Educación matemática, 26(3), 109-133.

Goodlander, G. (2008). Ghosts of a Chance Alternate Reality Game (ARG). Smithsonian American Art Museum. Recuperado de http://www.museumtwo.com/publications/Ghosts_Report. pdf

Gülseçen, S., Kararaş, E. \& Koçoğlu, F. (2012). Can Geogebra make easier the understanding of cartesian coordinates? a quantitative study in Turkey. International journal on new trends in education and their implications, 3(4). Recuperado de http:// www.ijonte.org/FileUpload/ks63207/ File/02.gulsecen.pdf

Kelly, W. A. (1982). Psicología de la educación. (8va ed.). Madrid: Morata.

Medina, M. y Rubio, H. (2013). Uso de software libre para el aprendizaje de la integral definida. Universidad Nacional de Rosario. Recuperado de http://ciaem-redumate.org/memoriasicemacyc/100-441-1-DR-C.pdf

Montero, M. (1987). Las prácticas de la enseñanza en la formación inicial: sentido curricular y profesional. En, La formación práctica de los profesores. Santiago: Tórculo.

Lit, W. (2013). The use of Geogebra in collaborative learning in mathematics. (Tesis de maestría). Universiti Teknologi Malaysia.

Olsson, J. (2017). GeoGebra, enhancing creative mathematical reasoning. (Tesis doctoral). Universidad en Umeå, Suecia. Recuperado de http:// urn.kb.se/resolve?urn=urn:nbn:se:u mu:diva-133050 
Oldfield, B. (1991). Games in the learning of mathematics part 1: a classification. Mathematics in School, 1(20), $41-43$.

Palmer, C. y Petroski A. (2016). Alternate Reality Games: gamification for performance. Pennsylvania: CRC Press.

Pérez, A., Zabalza, M. y Cid, A. (2009). Las prácticas de enseñanza declaradas de los mejores profesores de la Universidad de Vigo. Revista electrónica de investigación y evaluación educativa, 15 (2), 1-29.

Piñeiro, T. y Costa, C. (2015). ARG (juegos de realidad alternativa). Contribuciones, limitaciones y potencialidades para la docencia universitaria. Comunicar, 44(1), 141-148. https:// doi.org/10.3916/C44-2015-15

Pulido, P. (2002). Una estrategia didáctica para la enseñanza del álgebra lineal con el uso del sistema de cálculo algebraico Derive. Revista complutense de educación, 13(2), 645-675.

Talavera, R. y Marín, F. (2015). Recursos tecnológicos e integración de las ciencias como herramienta didáctica. Revista de Ciencias Sociales, 20(2). Disponible en https://www.redalyc. org/pdf/280/28041012011.pdf

Tobón, S., González, L., Nambo J. y Vázquez, J. (2015). La socioformación: un estudio conceptual. Paradigma, 36(1) 7-29. Recuperado de http://revistas. upel.edu.ve/index.php/paradigma/article/view/2661/1273

Tobón, S., Pimienta, J. y García, J. (2010). Secuencias didácticas: aprendizaje y evaluación de competencias. México, D.F.: Pearson.

Torres, M. (2010). La enseñanza tradicional de las ciencias versus las nuevas tendencias educativas. Educare, 14(1).
Waddington, D. (2013). A parallel world for the World Bank: A case study of urgent: Evoke, an educational alternate reality game. Revue internationale des technologies en pédagogie universitaire / International Journal of Technologies in Higher Education, 10(3), 42-56. http://doi.org/cqdj

Whitton, N. y Hollins, P. (2008). Collaborative virtual gaming worlds in higher education. ALT-J, 16(3), 221-229. http://doi.org/fknwcf 